\title{
PARCS: A Safety Net Community-Based Fitness Center for Low-Income Adults
}

\author{
NiCole Keith, PhD ${ }^{1,2,3}$, Deming Mi, MS $S^{4}$ Kisha Alexander, PhD ${ }^{5,6}$, Stephanie Kaiser, MS ${ }^{7}$, Mary de Groot, PhD ${ }^{8}$ \\ (1) Indiana University Center for Aging Research; (2) School of Physical Education and Tourism Management; (3) Regenstrief Institute, Inc.; (4) Division of Biostatistics, \\ Indiana University School of Medicine; (5) Accountable Health Solutions; (6) University of Phoenix; (7) National Institute of Fitness and Sport; (8) Diabetes Translational \\ Research Center, Indiana University School of Medicine \\ Submitted 18 September 2014, revised 17 Feb 2015, accepted 27 May 2015
}

Abstract

Background: Physical activity (PA) and fitness are critical to maintaining health and avoiding chronic disease. Limited access to fitness facilities in low-income urban areas has been identified as a contributor to low PA participation and poor fitness.

Objectives: This research describes community-based fitness centers established for adults living in low-income, urban communities and characterizes a sample of its members.

Methods: The community identified a need for physical fitness opportunities to improve residents' health. Three community high schools were host sites. Resources were combined to renovate and staff facilities, acquire equipment, and refer patients to exercise. The study sample included 170 members older than age 18 who completed demographic, exercise self-efficacy, and quality of life surveys and a fitness evaluation. Neighborhood-level U.S. Census data were obtained for comparison.

Results: The community-based fitness centers resulted from university, public school, and hospital partnerships offering safe, accessible, and affordable exercise opportunities. The study sample mean body mass index was $35+7.6 \mathrm{~kg} / \mathrm{m}^{2}$ (class II obesity), mean age was $50 \pm 12.5$ years, $66 \%$ were Black, $72 \%$ were female, $66 \%$ completed some college or greater, and $71 \%$ had an annual household income of less than $\$ 25,000$ and supported 2.2 dependents. Participants had moderate confidence for exercise participation and low fitness levels. When compared with census data, participants were representative of their communities.

Conclusion: This observational study reveals a need for affordable fitness centers for low-income adults. We demonstrate a model where communities and organizations strategically leverage resources to address disparities in physical fitness and health.

\section{Key Words}

Race, gender, age, exercise, disadvantaged, exercise, community-based participatory research, community health partnerships, community health research, health disparities, health outcomes, health promotion, delivery of health care, fitness centers, health priorities
A dults residing in low-income, inner-city communities are less likely to meet the U.S. Department of Health and Human Services recommended guidelines of 150 minutes of moderate to vigorous PA per week. ${ }^{1}$ Individual and environmental factors are associated with physical inactivity among low-income urban adults including physical, psychosocial and environmental (e.g., built environment) context. ${ }^{2-5}$ The absence of accessible and affordable resources to facilitate PA participation are known barriers within low-income urban communities when compared with more affluent areas. ${ }^{6-8}$ Research focused on PA participation and the overall health of inner-city community residents demonstrates that residents are more likely to be physically active when opportunities for PA are perceived as being relatively easy to access and suitable for their ability level..$^{9-12}$

Similar to "food deserts," where convenience stores are the primary food source and consumers' choices are limited to tobacco, liquor, and high-fat, processed foods, ${ }^{13-15}$ exercise des- 
erts can be characterized as built environments with limited walkability, access to green spaces, and/or affordable fitness facilities that promote PA and fitness among poor and underserved communities. ${ }^{16,17}$ In the greater Indianapolis area, as with many other American cities, GIS mapping demonstrates the geographic correlation between high obesity prevalence and poverty. Within the same geographic boundaries there are few exercise facilities that offer affordable memberships to residents with limited resources (Figure 1). Areas outlined in bold, red contain a population where more than $35 \%$ of the residents live in poverty and more than one-half are obese. Across studies, data suggest that to more completely attend to physical fitness-related health disparities, exercise outlets to address built environment deficiencies and access to affordable health-fitness professionals are needed in low-income neighborhoods. ${ }^{9111,18-21}$

Figure 1 also illustrates the YMCA locations serving the metropolitan service area of Indianapolis during the time of this study. Within the United States, the YMCA has a strong commitment to investing resources to improve community health. However, most YMCA locations in the metropolitan service area of Indianapolis would require that inner city residents travel 10 miles or more to the closest facility. In the Indianapolis area, like many other cities, public transportation to suburban communities is limited which makes access to these facilities difficult. Only two YMCA sites were centrally located and both were downtown, in a higher socioeconomic status area with lower obesity prevalence. Although the YMCA provides sliding scale income-based memberships, many low-income residents still cannot afford the fees. ${ }^{22-24}$ Improving the health and fitness of this community would require change by actions from a diverse group of community constituents and evidence that there is actually a community need for a fitness resource.

The current study uses a cross-sectional, observational design that describes community-based fitness centers established for adults living in low-income, urban neighborhoods and characterizes a sample of its members. We also present
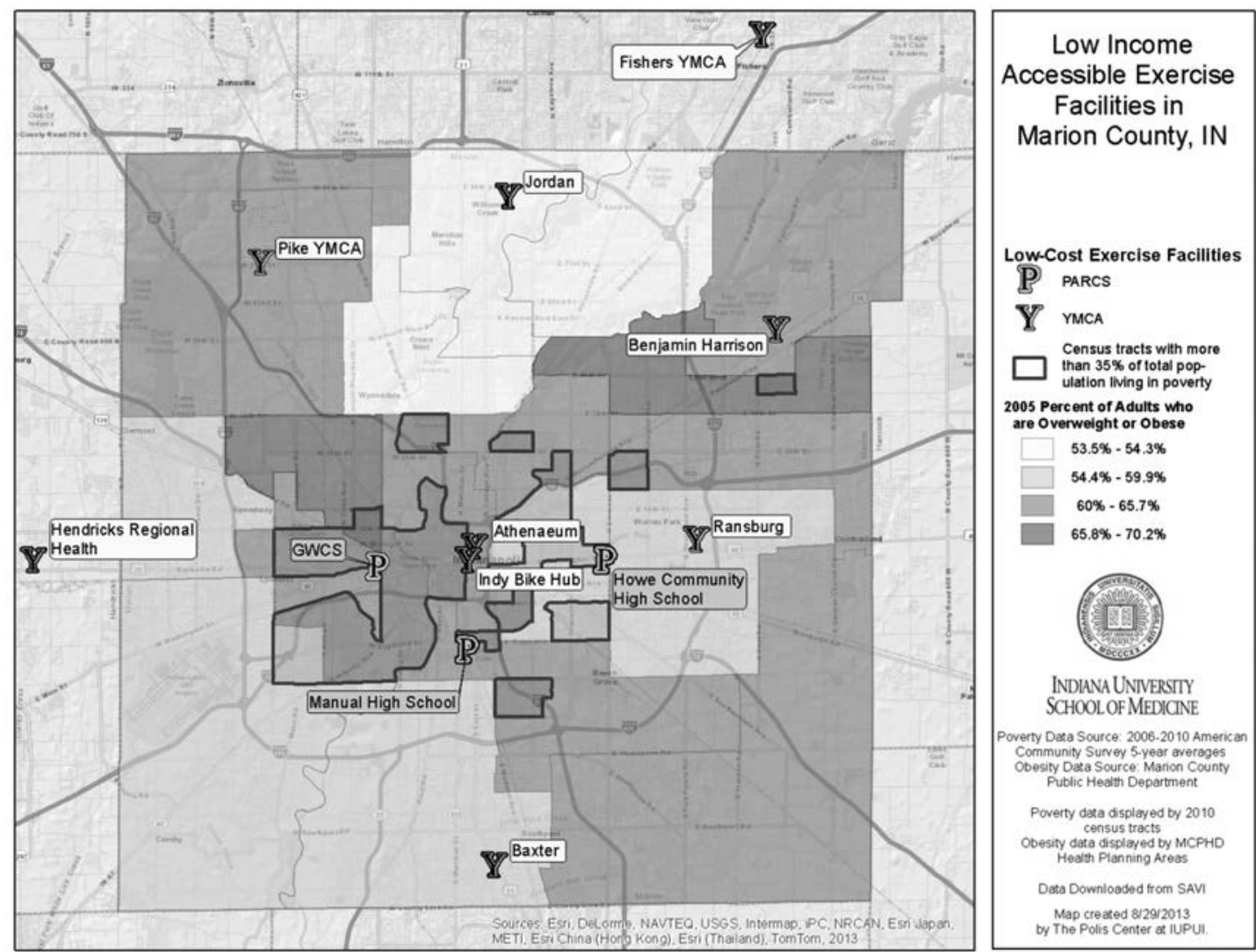

Figure 1. Prevalence of obesity, poverty and locations of affordable fitness facilities in the metropolitan service area of Indianapolis. 
U.S. Census data for all residents living in the communities that host these fitness centers and evaluate whether members were representative of their communities and whether these communities needed fitness centers.

\section{METHODS}

\section{Partnership}

To address limitations in accessible and affordable exercise opportunities as well as other needs, Physically Active Residential Communities and Schools (PARCS; formerly Fit for Life) was founded in 2001 as a result of a partnership (Figure 2) between the Indianapolis Public School (IPS) System, Indiana University-Purdue University, Indianapolis (IUPUI) Department of Kinesiology, and a weight management program located in Federally Qualified Health Centers (FQHCs) that are part of Indiana's largest public hospital system. ${ }^{25-28}$ The primary goals of PARCS are to 1) offer sustainable opportunities for community members to exercise in a facility that delivers sup- portive services, 2) provide teaching and learning opportunities for university faculty and students, and 3) provide primary care providers with fitness referral sites that are prepared to work with their patients. All partner sites were located within a 3-mile radius of IUPUI. The community was defined by the partner stakeholders and constituents who all lived and/or worked within the area highlighted in bold, red outline (Figure 1). In a community assessment, community leaders listed poor health as one major threat to community sustainability. ${ }^{28}$ At the same time, FQHC primary care providers were queried about the reasons their patients were not referred to exercise. Location, affordability, and the ability of a fitness professional to manage their patients with multiple comorbidities were listed reasons. ${ }^{29}$ Last, IUPUI Kinesiology students who were generally highly fit, young individuals from suburban and rural communities were training to be fitness and/or health professionals (many Kinesiology students go on to careers in hospital-based disease prevention and treatment or community health promotion). ${ }^{30}$ However, few IUPUI Kinesiology students had experience with

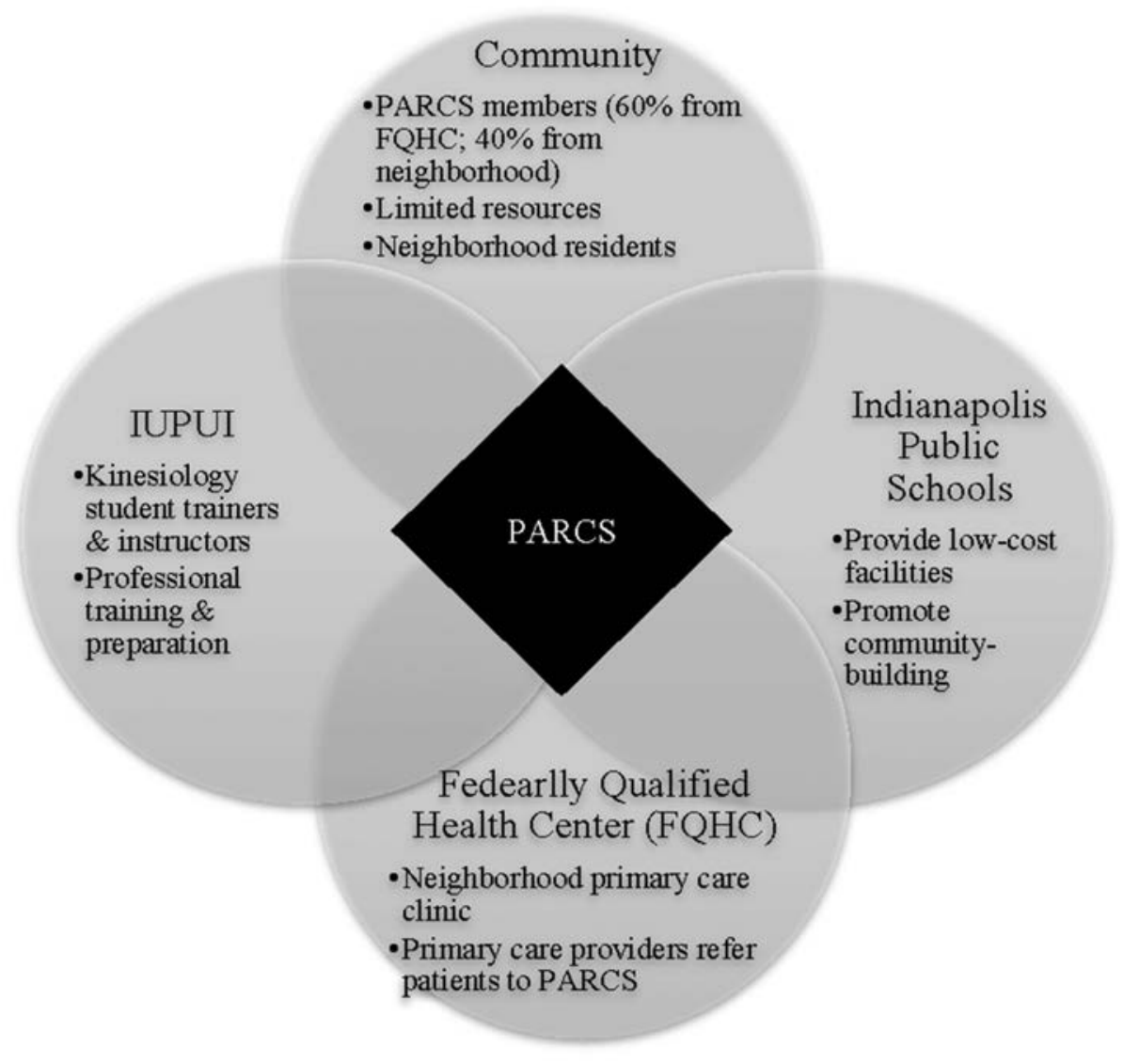

Figure 2. Community members' and partners' relationships and contributions. 
urban residents who had comorbid conditions, a population they would likely encounter during their professional careers. IUPUI faculty believed it was important to improve the skills and perceptions that Kinesiology students generally have regarding obese people..$^{31,32}$

PARCS was designed to create a series of safety net fitness facilities to meet the needs of all community partners. Similar to safety net FQHCs that provide health care for patients who are uninsured or underinsured, ${ }^{33}$ these safety net fitness facilities were conveniently located and provided access and services to members who could not afford regular or sliding scale, income-based fitness center memberships. From 2002 to 2009 there were three PARCS locations (shown in Figure 1): George Washington Community High School, Thomas Carr Howe Community High School, and Emmerich Manual High School. These IPS-designated "Community Schools" were already open to the community for shared use. IPS leaders indicated these sites would be best suited to host public fitness centers. Programming for adult community members occurred after school hours. IUPUI kinesiology students were supervised by faculty to serve as personal trainers and fitness instructors at each of the PARCS locations as they received professional preparation and academic course credit. Approximately 150 students enrolled across five academic classes each semester performed service learning activities at PARCS. Activities included health and fitness assessments, writing exercise prescriptions, creating exercise programs, leading group exercise, and/or offering personal training. Activities were tied to the objectives of the courses in which the kinesiology majors were enrolled. Cost-savings also occurred by using high school fitness facilities to house the program. Most fitness center equipment was accrued though grant funding acquired by the partners or through donations. PARCS membership fees of $\$ 20$ per year were collected by the host high schools to help maintain the facility and its equipment. In 2011, in response to school consolidation by IPS, PARCS moved to a new, larger, state-of-the art facility on IPS property near two of the previous high schools. PARCS continues to operate in one of its original high school locations and more than 3,000 people have joined over a 13-year span. School, community leaders, PARCS members, and FQHC providers continue to advise PARCS leaders about programming and student staffing. The PARCS Program
Director also attends regular community meetings. Partners pooled resources to establish PARCS as a community asset that could address their separate problems.

In this paper, we characterize PARCS members through demographic, PA, exercise self-efficacy, quality of life, health, and physical fitness data for a sample of PARCS members. Study protocol was determined by community partners who agreed that characterizing the PARCS program would require both qualitative and quantitative data. These data were obtained simultaneously and only quantitative data are presented in this report. Study approval was obtained from the Indiana University Institutional Review Board by the faculty investigators. The principal investigators trained eight research assistants (RAs) to collect both demographic and physical data. RAs participated in quarterly refresher training sessions and met investigators weekly to discuss study progress over an 18-month period. Over this period, all PARCS members over age 18 were sent a letter requesting their participation in the research study. We sent letters in waves of 200 and called members up to three times or until we received a response. Once the wave was complete, another group of letters were mailed until all members for whom we had addresses were sent a letter. Flyers were placed at PARCS locations and distributed by RAs to members. Members were asked to call a local study phone number to receive additional information and enroll in a research study.

Members who agreed to participate in the research received instructions when their appointment was scheduled with a trained RA at a PARCS site. Instructions were delivered again during a reminder phone call that occurred the day before the scheduled test. Testing occurred during regular fitness center hours (between 4:00 PM and 7:00 PM) at a time when members were available for an appointment. Upon arrival to their scheduled appointment, participant informed consent was obtained by the RA. All measures were performed in a single visit and took approximately 90 minutes to complete. Participants received \$20 after the visit.

\section{Measures to Support the Community Need}

Surveys evaluated demographics, health history, exercise self-efficacy, PA participation, and quality of life. All survey measures were led and participant responses were recorded by a RA who also was a trained interviewer. Immediately after 
survey completion, physical testing was performed.

Demographics and Health History. Participants provided demographic information such as date of birth, highest completed grade, and annual household income. The Health History Questionnaire asked participants to indicate the presence or absence of cardiovascular disease risk factors (e.g., family history, smoking, hypertension). The number of risk factors participants reported at present were summed for analyses with a range of zero to six.

Exercise self-efficacy was measured using the 18-item Exercise Self-Efficacy Scale to assess confidence in engaging in exercise in the context of 6 common barriers (negative affect, excuse making, exercising alone, lack of access, resistance from others, and bad weather) on a 5-point Likert scale from 1 [not at all confident], 2 [somewhat confident]; 3 [moderately confident]; 4 [very confident]; to 5 [completely confident]. The measure has been found to have adequate internal consistency ( $\alpha=0.76$ to 0.82 ) and good construct validity ${ }^{34-36}$ and has good internal validity and adequate external validity in NonHispanic Blacks with a coefficient $\alpha$ of 0.80 and factor loadings from each of the six barriers ranging from 0.49 to $0.70 .{ }^{37}$

Yale Physical Activity Scale. The Yale Physical Activity Scale is a comprehensive survey that measures PA behaviors performed in the past month across various intensity levels and in multiple domains including leisure and vigorous walking; heavy housework and yard work; jogging and playing basketball; climbing stairs; standing and moving around; standing without moving around; and sitting. Summary score indices were determined for each domain (vigorous activity, leisure walking, moving, standing, and sitting). The Yale Physical Activity Scale has relatively good test-retest reliability with correlation coefficients ranging between 0.42 and $0.65 .{ }^{38}$ For this study, the five activity dimensions were scored (adjusted for intensity of activity) and summed to create an Activity Dimension Summary Index, expressed as total units for each participant to summarize activity levels and intensity for the past month. Scores ranged from 0 to 145 and higher scores indicate more activity. Other studies have used this tool in a variety of age and race groups and have found good validity and reliability. ${ }^{39}$

Short Form-36 Quality of Life. The Short Form HealthRelated Quality of Life survey was administered as an overall health-related quality of life measure. The 36 items evaluate 8 domains of health: physical functioning (ability to engage in physical activities), role-physical (impact of physical health on role-based activities), general health perception (global assessment of health), vitality (energy, fatigue), social functioning (physical or emotional health impact on social activities), role-emotional (impact of emotional health on role performance) and mental health (depression, anxiety). Scales are calculated on a 100-point scale where higher scores indicate more favorable levels of functioning. ${ }^{22}$ In addition, physical component scores and mental component scores, derived from all of the domain subscales, were calculated and standardized on a 100-point scale with 50 as the median, and normed using 1998 U.S. Census data.

\section{Health and Fitness Measures Occurred in the Following Order}

Anthropometric measures. Height and weight were measured using a wall-mounted stadiometer and a calibrated digital scale to the nearest pound. Calibration of the scales was performed quarterly or as needed. body mass index was calculated post hoc using a formula [weight $\left.(\mathrm{kg}) / \mathrm{height}(\mathrm{m})^{2}\right]$.

Resting heart rate and blood pressure. Resting heart rate was palpated from the participant's left radial artery (unless otherwise instructed by the participant). The number of beats were counted for 15 seconds, and then multiplied by four. Resting blood pressure was measured on the left arm (unless otherwise instructed by the participant) using a manual sphygmomanometer and stethoscope.

Muscular strength and endurance. Muscular strength and endurance were measured through the partial curl-up test based on the American College of Sports Medicine protocol. ${ }^{40}$ Participants performed as many repetitions as possible in 1 minute without stopping (maximum of 25 repetitions). The test was terminated if the cadence of 40 beats/min was broken and only full repetitions were counted. Scores range from 9 to 25 and the classifications include "needs improvement," "fair," "good," "very good," and "excellent." A higher score is a better score. ${ }^{40}$

Muscular flexibility. Lower body flexibility was assessed using the chair sit and reach protocol. ${ }^{41}$ The participant was instructed to bend one leg while straightening the other and to reach down the straight leg as far as possible, holding this position approximately 2 seconds. The score was recorded as the most distant point (in centimeters) from the fingertip to the toe. The test is scored as follows: less than 0.5 is below average, 
0.5 to 6.5 is average, and greater than 6.5 is above average. ${ }^{42}$

Upper body flexibility was assessed using the back scratch protocol. ${ }^{43}$ The final score was recorded to the nearest one-half inch, measuring the distance of overlap or distance between the tips of the middle fingers. A negative score was given if the middle fingers did not touch, a zero if middle fingers touched and a positive score if the middle fingers overlapped. The test is scored as follows: less than -1 is below average, -1 to 3 is average, and greater than 3 is above average. ${ }^{42}$

U.S. Census data. Data from the 2010 U.S. Census were gathered in collaboration with the IUPUI Polis Center data system to provide a sociodemographic comparison of PARCS members and neighborhood residents living in the areas where PARCS sites were located. Demographic data were drawn at the census tract level. Geocoding was used to identify the census tracts where study participants lived.

\section{Statistical Analyses}

Analyses were conducted using R (R Core Team, 2013). Continuous variables were summarized with mean and stan-

Table 1. Demographic Characteristics of Physically Active Residential Communities and Schools (PARCS) Participants and Neighborhood Census

\begin{tabular}{|c|c|c|c|c|c|c|c|}
\hline \multirow[b]{2}{*}{ Characteristic } & \multicolumn{2}{|c|}{$\begin{array}{l}\text { PARCS Participants } \\
\qquad(N=170)\end{array}$} & \multirow{2}{*}{$\begin{array}{l}2010 \text { U.S. } \\
\text { Census } \\
\text { Tract Data } \\
(\%)\end{array}$} & \multirow[b]{2}{*}{ Characteristic } & \multicolumn{2}{|c|}{$\begin{array}{l}\text { PARCS Participants } \\
\qquad(N=170)\end{array}$} & \multirow{2}{*}{$\begin{array}{c}2010 \text { U.S. } \\
\text { Census } \\
\text { Tract Data } \\
(\%)\end{array}$} \\
\hline & $n$ & $\%$ & & & $n$ & $\%$ & \\
\hline \multicolumn{4}{|l|}{ Age, y $(49.9 \pm 12.5)$} & \multicolumn{4}{|l|}{ Home ownership } \\
\hline $18-44$ & 53 & 31 & 48 & Yes & 69 & 42 & 41 \\
\hline $45-64$ & 97 & 57 & 33 & \multicolumn{4}{|l|}{ Work outside of the home } \\
\hline$\geq 65$ & 20 & 12 & 11 & Yes & 88 & 52 & 85 \\
\hline \multicolumn{4}{|l|}{ Gender } & No. of dependents (mean, $S D$ ) & 2.2 & 1.3 & \\
\hline Male & 48 & 28 & 48 & Difficulty making ends meet & & & \\
\hline Female & 122 & 72 & 52 & Very hard & 43 & 25 & \\
\hline \multicolumn{4}{|l|}{ Race } & Hard & 42 & 25 & \\
\hline White & 57 & 34 & 33 & Not hard, not easy & 68 & 40 & \\
\hline Black & 113 & 66 & 49 & Easy & 13 & 8 & \\
\hline \multicolumn{4}{|l|}{ Marital status } & Very easy & 4 & 2.4 & \\
\hline Married & 69 & 41 & 36 & \multicolumn{4}{|l|}{ Difficulty paying bills } \\
\hline Widowed & 13 & 8 & 7 & Yes & 75 & 45 & \\
\hline Divorced & 43 & 25 & 15 & \multicolumn{4}{|l|}{ Financial situation in the past year } \\
\hline Separated & 9 & 5 & - & Getting better & 48 & 29 & \\
\hline Never married & 36 & 21 & 43 & Staying the same & 90 & 54 & \\
\hline \multicolumn{4}{|l|}{ Education } & Getting worse & 29 & 17 & \\
\hline \multirow{2}{*}{$\begin{array}{l}\text { Without high school } \\
\text { diploma }\end{array}$} & \multirow[t]{2}{*}{13} & \multirow[t]{2}{*}{8} & \multirow[t]{2}{*}{25} & \multicolumn{4}{|l|}{ Financial satisfaction } \\
\hline & & & & Very satisfied & 5 & 3 & \\
\hline High school diploma only & 42 & 26 & 36 & Somewhat satisfied & 27 & 16 & \\
\hline Some college or trade school & 56 & 34 & 19 & \multirow{2}{*}{$\begin{array}{l}\text { Neither satisfied or } \\
\text { dissatisfied }\end{array}$} & \multirow[t]{2}{*}{46} & \multirow[t]{2}{*}{27} & \\
\hline Associate degree or greater & 53 & 32 & 19 & & & & \\
\hline \multicolumn{4}{|l|}{ Income (\$U.S.) } & \multirow{2}{*}{$\begin{array}{l}\text { Somewhat dissatisfied } \\
\text { Very dissatisfied }\end{array}$} & 56 & 33 & \\
\hline$<25,000$ & 120 & 71 & 41 & & 34 & 20 & \\
\hline $25,000-49,000$ & 34 & 20 & 32 & \multicolumn{4}{|l|}{ Hopeful about financial situation } \\
\hline $50,000-74,999$ & 8 & 5 & 16 & Pretty hopeful & 91 & 54 & \\
\hline \multirow[t]{2}{*}{$>75,000$} & 6 & 4 & 13 & More or less hopeful & 65 & 39 & \\
\hline & & & & Not hopeful at all & 11 & 7 & \\
\hline
\end{tabular}


dard deviation. Categorical variables were summarized using frequency counts and percent. Variables were assessed for normality and heteroskedasticity.

\section{RESULTS}

Partner-generated financial support for this research established a recruitment goal of 200 members over an 18 -month time period. We reached $85 \%$ of that goal and a total sample of 170 members from the three PARCS locations volunteered for this study. All participants completed all measures. Demographic characteristics are shown in Table 1. Across the sites, the mean age was $49.9 \pm 12.5$ years. Sixty-six percent of the sample self-identified as African American or Black, $72 \%$ were female, $64 \%$ reported completion of some college or greater, $71 \%$ reported an annual household income of less than $\$ 25,000$ with an average of $2.2 \pm 1.3$ dependents, $51 \%$ percent reported working outside the home, and 50\% reported considerable financial strain.

Exercise self-efficacy results are presented in Table 2.
Findings indicated moderate confidence in participants' ability to engage in exercise in the context of a variety of common barriers (e.g., exercising alone, lack of access, resistance from others).

Scores for the Yale Physical Activity Scale activity dimension indices (Table 2) show that the population had a moderate level of engagement in overall physical activities with a mean score of $52.2 \pm 28.3$ out of a possible score of 145 . Participants showed high levels of engagement in vigorous activities with a mean score of $28.8 \pm 20.7$ out of a possible score of 60 and reported less engagement in lower intensity exercises (moving, sitting, and standing).

Evaluation of health-related quality of life (Short Form -36) domains (Table 2) indicated that the highest ratings were observed in social functioning (impact of physical and emotional health on social functioning such as social isolation associated with limitations to physical mobility) and roleemotional functioning (impact of emotional health on role performance such as the impact of depression on ability to work). The mean score for the physical component scores

\begin{tabular}{|c|c|c|c|}
\hline Characteristics & Mean & $S D$ & Possible Range \\
\hline \multicolumn{4}{|l|}{ Psychosocial } \\
\hline Exercise self-efficacy & 3.3 & 0.9 & $0-5$ \\
\hline \multicolumn{4}{|l|}{ Short Form-36 survey } \\
\hline Physical functioning & 78.2 & 27.4 & $0-100$ \\
\hline Role physical & 73.5 & 38.9 & $0-100$ \\
\hline General health perception & 69.2 & 19.8 & $0-100$ \\
\hline Mental health score & 76.7 & 16.9 & $0-100$ \\
\hline Vitality & 62.4 & 19.8 & $0-100$ \\
\hline Social functioning & 82.3 & 22.3 & $0-100$ \\
\hline Role emotional & 73.4 & 38.9 & $0-100$ \\
\hline Physical component score & 47.7 & 11.1 & $0-100$ \\
\hline Mental component score & 51.3 & 9.9 & $0-100$ \\
\hline \multicolumn{4}{|c|}{ Yale Physical Activity Scale, activity dimensions index } \\
\hline Summary index & 52.2 & 28.3 & $0-145$ \\
\hline Vigorous activity index & 28.8 & 20.7 & $0-60$ \\
\hline Leisure walking index & 10.5 & 8.3 & $0-48$ \\
\hline Moving index & 10.5 & 4.4 & $0-18$ \\
\hline Standing index & 3.6 & 2.7 & $0-15$ \\
\hline Sitting index & 2.4 & 1.0 & $0-4$ \\
\hline
\end{tabular}


(summary score for physical functioning) fell between the 25th and 50th percentiles for the general U.S. population.

The mean score for the mental component scores (summary score for mental health functioning) was just below the 50th percentile for the general U.S. population.

Health and fitness indices. Fitness evaluation data are presented in Table 3. The average number of self-identified cardiovascular disease risk factors was $2.0 \pm 1.6$. This sample of fitness center members was generally obese with an average body mass index of $35.0 \pm 7.6 \mathrm{~kg} / \mathrm{m}^{2}$. The average resting heart rate indicated below average cardiovascular fitness. The mean systolic blood pressure revealed prehypertension, whereas the diastolic blood pressure was within normal range. Partial curl up scores yielded a "good" classification..$^{40}$ Participants also demonstrated below average lower body flexibility (chair sit and reach test) and average upper-body flexibility (back scratch test)..$^{41,44}$

U.S. Census comparisons. U.S. Census tract data from 2010 were examined for 20 neighborhood-level tracts where the largest number of study participants lived (Table 1). All tracts reported a roughly even gender distribution. Examination of racial proportion demonstrated that $48.8 \%$ of community residents were African American, 61\% had a high school diploma or lower, $41 \%$ had an income of less than $\$ 25,000$, and $85 \%$ of the population residing within these census tracts worked outside of the home.

\begin{tabular}{|c|c|c|}
\hline \multicolumn{3}{|c|}{$\begin{array}{l}\text { Table 3. Health and Fitness Characteristics of } \\
\text { Physically Active Residential Communities and } \\
\text { Schools Participants }(N=170)\end{array}$} \\
\hline Characteristics & Mean & SD \\
\hline \multicolumn{3}{|l|}{ Health/physical } \\
\hline No. of cardiovascular disease risk factors & 2.0 & 1.60 \\
\hline Body mass index & 35.0 & 7.60 \\
\hline Weight (kg) & 96.6 & 22.30 \\
\hline \multicolumn{3}{|l|}{ Fitness characteristics } \\
\hline Resting heart rate (beats/min) & 76.7 & 12.20 \\
\hline Resting systolic blood pressure (mm Hg) & 125.8 & 13.00 \\
\hline Resting diastolic blood pressure (mm Hg) & 76.6 & 8.60 \\
\hline Partial curl-up (total number) & 11.5 & 10.20 \\
\hline Sit-and-reach test $(\mathrm{cm})$ & 2.2 & 10.12 \\
\hline Back scratch test $(\mathrm{cm})$ & -10.3 & 15.60 \\
\hline
\end{tabular}

\section{Results Summary}

Objectives of this research included characterizing the health and fitness of PARCS members and barriers that would prevent community member access to a fitness center without the existence of PARCS. Study results and U.S. Census data (Figure 1) show PARCS members who agreed to participate in this study and residents living in the communities surrounding PARCS sites both demonstrate a need for affordable fitness options due to their economic situation and physical condition. Figure 1 shows that PARCS was established in communities where, according to U.S. Census data, 35\% of the population lives in poverty and $53.5 \%$ to $70.2 \%$ are overweight or obese, depending on the neighborhood. Research participants were generally obese with 2 cardiovascular disease risk factors and moderate confidence in their ability to exercise in the face of barriers. Cost is a recognized barrier to exercising in a fitness facility; $71 \%$ of the participants reported an income of less than $\$ 25,000,50 \%$ reported high levels of financial strain, and $40 \%$ reported moderate levels of financial strain. Results support a demonstrated need for financially and geographically accessible fitness programs.

\section{DISCUSSION}

These data reveal one of the few presentations of demographics, quality of life, PA participation, exercise self-efficacy, and physical fitness among a low-income sample of members who joined a safety net fitness center. Organizations (a public school system, a university, and FQHCs) have collaborated to offer sustainable public exercise options for more than 13 years. Public schools can be used to promote adult PA, provide diverse clients to support university student learning, and offer affordable and accessible sites where providers can send their patients with comorbidities to exercise safely. Partners worked to generate funding, equipment, staff, and community member support to create a program that fulfilled their mutual and self-interests. PARCS was formed and sustained during economically uncertain times, especially for IPS. The enduring commitment to community health by multiple institutions serves as a model for what is possible in other communities where goals are shared and prioritized across institutions.

A key finding of this research is the majority of participants had limited discretionary income to pay for a regular 
or sliding scale fitness center membership. Research suggests that greater life demands on low-income populations coupled with limited access to fitness facilities negatively influence PA participation. ${ }^{8,16,22-24}$ Including PA within the community needs assessment was an important facilitator of this partnership. Both policies and planning for safety net fitness centers such as PARCS could aid in addressing low physical fitness and perhaps, eventually reduce health disparities for residents of low-income communities.

Self-report data revealed that, on average, study participants had two cardiovascular disease risk factors. Measured data showed participants had high resting heart rates (indicating poor cardiovascular health), were prehypertensive, generally obese, and demonstrated generally moderate or low fitness in nearly every measured category. Our sample represented groups (e.g., low-income, racial-ethic minority, living in underresourced communities) who have a higher incidence of obesity and are at greater risk for cardiovascular disease. ${ }^{45,46}$ Additionally, $56 \%$ of our sample received a FQHC medical referral. In 2012, the Institute of Medicine recommended health care providers perform routine behavior assessments including PA screening. It also was recommended that providers advocate for PA resources for their patients and within the communities where their patients live. ${ }^{21}$ Although PARCS existed long before the Institute of Medicine recommendation, the partnership and program are consistent with the Institute of Medicine guidelines.

This observational, cross-sectional study designed to characterize the demographic, psychological and fitness characteristics of these sites had several limitations. PARCS was developed to serve rather than study its members. We learned to propose research only after a strong partnership was established. Permission to present all assessment results was not a membership requirement. Also, many phone numbers were no longer in service and mail was returned with no forwarding address; thus, obtaining retrospective consent to present all member comparison data was not possible. Instead, we present U.S. Census data to demonstrate that residents living in the communities surrounding PARCS were similar when compared with study participants. IPS Schools collected membership fees but did not record join dates so there is no record of the length of time any members, including study participants, were PARCS members. Health and fitness data show that the members who volunteered for this research were obese with two or more self-reported cardiovascular disease risk factors and on average were prehypertensive (based on measured resting blood pressure). This demonstrates there remains a need for this group to exercise regardless of whether their health status was impacted positively by in engaging in PA at PARCS. There was no attempt to apply culturally specific approaches to members. Rather, the program was tailored to the needs and preferences of individual participants. We attempted to evaluate cardiorespiratory response to exercise (e.g., exercise heart rate and blood pressure); however, owing to the fitness center's environment (loud music, several machines being used simultaneously, and people talking) the data that we received were deemed unreliable and we only include resting cardiorespiratory measures in this report. Here we report one portion of this research study. The entire evaluation also included qualitative data collection of members who volunteered for the study, faculty and students as well as key informant interviews of community stakeholders. These data will be presented in future reports.

Efforts to increase the reach and participation levels of community-based programs will require a combination of individual and program level focus. We found that community members with generally low physical fitness will make use of an accessible fitness resource despite financial barriers and poor health status. Particular deficiencies in health and physical fitness within health disparate populations could be addressed by creating affordable, accessible exercise opportunities. Such efforts require open, honest, purposeful, and continued communication among partners who are trusting of and willing to make adjustments with leaders and constituents from each group. PARCS serves as one model where communities and organizations leverage their resources through strategic collaboration to address the needs of urban residents living within exercise deserts.

\section{ACKNOWLEDGMENTS}

This project was supported by the IUPUI Center for Service and Learning, IUPUI Office of Neighborhood Partnerships, Anthem Blue Cross and Blue Shield Foundation, IUPUI Vice Chancellor for Research Office and NIDDK Grant DK018092765. Census data were provided by the Indiana University Polis Center collected via SAVI. We thank Jay 
Colbert and Karen Fredericks Comer for their collaboration

in gathering these data.

The authors thank the Jim Grim of IPS for identifying key community stakeholders that developed and continue to support PARCS. We thank PARCS study participants for informing this research and all PARCS members who continue

\section{REFERENCES}

1. Committee PAGA. Physical activity guidelines advisory committee report, 2008. Washington (DC): U.S. Department of Health and Human Services; 2008.

2. Sallis JF, Floyd MF, Rodríguez DA, et al. Role of built environments in physical activity, obesity, and cardiovascular disease. Circulation. 2012;125(5):729-37.

3. Van Cauwenberg J, De Bourdeaudhuij I, De Meester F, et al. Relationship between the physical environment and physical activity in older adults: a systematic review. Health Place. 2011;17(2):458-69.

4. Saelens BE, Sallis JF, Frank LD, et al. Neighborhood environment and psychosocial correlates of adults' physical activity. Med Sci Sports Exerc. 2012;44(4):637-46.

5. Artinian NT, Fletcher GF, Mozaffarian D, et al. Interventions to promote physical activity and dietary lifestyle changes for cardiovascular risk factor reduction in adults a scientific statement from the American Heart Association. Circulation. 2010;122(4):406-41.

6. Gordon-Larsen $\mathrm{P}, \mathrm{Nelson} \mathrm{MC}$, Page $\mathrm{P}$, et al. Inequality in the built environment underlies key health disparities in physical activity and obesity. Pediatrics. 2006;117(2):417-24.

7. Moore LV, Diez Roux AV, Evenson KR, et al. Availability of recreational resources in minority and low socioeconomic status areas. Am J Prev Med. 2008;34(1):16-22.

8. Lee RE, Cubbin C, Winkleby M. Contribution of neighbourhood socioeconomic status and physical activity resources to physical activity among women. J Epidemiol Community Health. 2007;61(10):882-90.

9. Bennett GG, McNeill LH, Wolin KY, et al. Safe to walk? Neighborhood safety and physical activity among public housing residents. PLoS Med. 2007;4(10):e306.

10. Bopp M, Fallon E. Community-based interventions to promote increased physical activity: a primer. Appl Health Econ Health Policy. 2008;6(4):173-87.

11. Floyd MF, Taylor WC, Whitt-Glover M. Measurement of park and recreation environments that support physical activity in low-income communities of color: Highlights of challenges and recommendations. Am J Prev Med. 2009;36 Suppl 4:S156-60.

12. Yancey AK, McCarthy WJ, Harrison GG, et al. Challenges in improving fitness: results of a community-based, randomized, controlled lifestyle change intervention. J Womens Health (Larchmt). 2006;15(4):412-29. to help faculty and students teach and learn. We thank Eskenazi Health providers for their support of IUPUI faculty and student teaching and learning. In addition, we wish to thank student RAs Kyle McIlrath, Deia Davis, Timothy Graves, Mitchell Arthur, Lakenvia Ledford, LeAndra Brown, Sarah Herrera, and Elizabeth Counsell for their assistance in data collection.

13. Walker RE, Keane CR, Burke JG. Disparities and access to healthy food in the United States: A review of food deserts literature. Health Place. 2010;16(5):876-84.

14. Harwood EM, Erickson DJ, Fabian LE, et al. Effects of communities, neighborhoods and stores on retail pricing and promotion of beer. J Stud Alcohol Drugs. 2003;64(5):720.

15. Toomey TL, Chen V, Forster JL, et al. Do cigarette prices vary by brand, neighborhood, and store characteristics? Public Health Rep. 2009;124(4):535.

16. Estabrooks PA, Lee RE, Gyurcsik NC. Resources for physical activity participation: does availability and accessibility differ by neighborhood socioeconomic status? Ann Behav Med. 2003;25(2):100-4.

17. Powell LM, Slater S, Chaloupka FJ, et al. Availability of physical activity-related facilities and neighborhood demographic and socioeconomic characteristics: A national study. J Info. 2006;96(9).

18. Sallis JF, Millstein RA, Carlson JA. for Physical activity. Making healthy places: Designing and building for health, well-being, and sustainability. Washington (DC): Island Press/ Center for Resource Economics;2011:33.

19. Sallis R. Exercise is medicine and physicians need to prescribe it! Br J Sports Med. 2009;43(1):3-4.

20. Sallis JF, Glanz K. Physical activity and food environments: solutions to the obesity epidemic. Milbank Q. 2009;87(1):123-54.

21. Institute of Medicine. Accelerating progress in obesity prevention; Solving the weight of the nation. Washington (DC). 2012 [cited 2012 May 9]. Available from: www.iom.edu/Reports/2012 /Accelerating-Progress-in-Obesity-Prevention.aspx

22. Kirchhoff AC, Elliott L, Schlichting JA, et al. Strategies for physical activity maintenance in African American women. Am J Health Behav. 2008;32(5):517.

23. Azar D, Naughton GA, Joseph CW. Physical activity and social connectedness in single-parent families. Leisure Stud. 2009;28(3):349-58.

24. Silva $\mathrm{M}$, Cashman $\mathrm{S}$, Kunte $\mathrm{P}$, et al. Improving population health through integration of primary care and public health: providing access to physical activity for community health center patients. Am J Public Health. 2012;102(11):e56-e61.

25. Clark DO, Keith N, Chrysler L, et al. Design and reach of a primary care weight management program. J Health Care Poor Underserv. 2008;19(1):171-9. 
26. Clark D, Chrysler L, Perkins A, et al. Screening, referral, and participation in a weight management program implemented in five CHCs. J Health Care Poor Underserv. 2010;21(2):617-28.

27. Keith NR, Russell JA. Creating a climate of organizational diversity: Models of best practice. Kinesiol Rev. 2013;2:190-202.

28. Bringle RG, Officer SD, Grim J, et al. George Washington community high school: Analysis of a partnership network. New Directions for Youth Development. 2009;2009(122):41-60.

29. Keith NR, Clark DO, Perkins A. Contact Frequency and Weight Loss in a Primary Care-Based Weight Management Program: 997: May 30 4:15 PM-4:30 PM. Med Sci Sports Exerc. 2008;40(5).

30. Gledhill NS, Jamnik R. Careers opportunities for exercise science/kinesiology graduates. Health \& Fitness Journal of Canada. 2009;2(1):25-8.

31. Rukavina PB, Li W, Rowell MB. A service learning based intervention to change attitudes toward obese individuals in kinesiology pre-professionals. Soc Psychol Educ. 2008;11(1):95-112.

32. Hemingson D, Shim A, Choi H-s. Does a professor's somatotype influence perceptions of their intelligence from exercise science students? J Sports Sci. 2013;1:46-51.

33. Forrest $\mathrm{CB}$, Whelan E-M. Primary care safety-net delivery sites in the United States: A comparison of community health centers, hospital outpatient departments, and physicians' offices. JAMA. 2000;284(16):2077-83.

34. Benisovich SV, Rossi JS, Norman GJ, et al. Development of a multidimensional measure of exercise self-efficacy. Ann Behav Med. 1998;20 Suppl:S190.

35. Prochaska JO, Marcus BH. The Transtheoretical Model: applications to exercise. Advances in Exercise Adherence. Champaign (IL): Human Kinetics; 1994. p. 161-80.

36. Marcus BH, Owen N. Motiational readiness, self-efficacy and decision making for exercise. J Appl Soc Psychol. 1992;22:3-16.
37. Blaney CL, Robbins ML, Paiva AL, et al. Validation of the measures of the transtheoretical model for exercise in an Adult African-American sample. Am J Health Promot. 2012;26(5):317-25.

38. Dipietro L, Caspersen CJ, Ostfeld AM, et al. A survey for assessing physical activity among older adults. Med Sci Sports Exerc. 1993:628-42.

39. Young DR, Jee SH, Appel LJ. A comparison of the Yale Physical Activity Survey with other physical activity measures. Med Sci Sports Exerc. 2001;33(6):955-61.

40. American College of Sports Medicine. ACSM's guidelines for exercise testing and prescription: 8th edition. Philadelphia: Lippincott Williams \& Wilkins; 2010.

41. Rikli R, Jones J. American College of Sports Medicine: Senior fitness test manual. Wright JP, Cole A, editors. Champaign (IL): Human Kinetics; 2001: 43.

42. Rikli RE, Jones CJ. Senior fitness test manual. Champaign (IL): Human kinetics; 2013

43. Gordon NF. ACSM's guidelines for exercise testing and prescription. Philadelphia: Lippincott Williams \& Wilkins; 2009.

44. Pescatello LS, Riebe D. ACSM's Guidelines for Exercise Testing and Prescription the Ninth Edition-A Preview. Baltimore: Lippincott Williams \& Wilkins;2013.

45. Mathieu RAt, Powell-Wiley TM, Ayers CR, et al. Physical activity participation, health perceptions, and cardiovascular disease mortality in a multiethnic population: the Dallas Heart Study. Am Heart J. 2012;163(6):1037-40.

46. Weiss DR, O'Loughlin JL, Platt RW, et al. Five-year predictors of physical activity decline among adults in low-income communities: A prospective study. Int J Behav Nutr Phys Activ. 2007;4(1):2. 